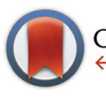

CrossMark click for update

Cite this: Org. Biomol. Chem., 2015, 13,8067

\title{
Functionalising the azobenzene motif delivers a light-responsive membrane-interactive compound with the potential for photodynamic therapy applications $\uparrow$
}

\author{
Theodore J. Hester, ${ }^{a}$ Sarah R. Dennison, ${ }^{b}$ Matthew J. Baker ${ }^{c}$ and Timothy J. Snape ${ }^{\star b}$ \\ When adorned with $n$-octyl chains azobenzene is able to disrupt a variety of calcein-loaded phospholipid \\ liposomes. The levels of lysis observed are dependent both on the lipid headgroup and the conformation \\ of the azobenzene compound. In all cases studied, it has been shown that the cis-conformer is more \\ membrane-interactive than the trans-conformer, suggesting that this class of molecule could be \\ optimised for photo-dynamic therapy applications against infectious pathogens.
}

Received 9th March 2015,

Accepted 25th June 2015

DOI: $10.1039 / c 50 b 00465 a$

www.rsc.org/obc

\section{Introduction}

The use of light for the photoisomerisation of azobenzene (1, Scheme 1) and its derivatives has been exploited in numerous biomedical applications. ${ }^{1}$ For example, light as a trigger has been used to induce changes in a molecular assembly for the controlled release of chemical compounds; ${ }^{2}$ to drive functional changes in peptides, proteins, nucleic acids, lipids, and carbohydrates; ${ }^{3}$ and for the in vivo light-activation of ion channels for the remote control of neuronal firing which has potential applications in controlling activity downstream from sites of neural damage or degeneration. ${ }^{4}$ Moreover, proteasome inhibitors, widely used as cytotoxic agents in cancer treatment, have been prepared through the introduction of a photoswitchable azobenzene motif into the molecular structures studied, giving rise to chemotherapeutic agents that can be switched on and off with light. ${ }^{5}$

The precedent for azobenzene derivatives to be exploited as light-triggered molecular switches in biological systems suggested that such a practice might be combined with our current interests studying the nature of non-covalent interactions between conformationally distinct benzanilides (e.g. 2) and diarylureas with phospholipid monolayers, ${ }^{6-9}$ if suitable changes to the azobenzene motif could be incorporated, as a

\footnotetext{
${ }^{a}$ School of Forensic and Investigative Sciences, University of Central Lancashire, Preston, PR1 2HE, UK

${ }^{b}$ School of Pharmacy and Biomedical Sciences, University of Central Lancashire, Preston, PR1 2HE, UK. E-mail: tjsnape@uclan.ac.uk; Tel: +44 (0)1772 895805 ${ }^{c}$ WestCHEM, Department of Pure and Applied Chemistry, University of Strathclyde, Glasgow, G1 1XL, UK. E-mail: matthew.baker@strath.ac.uk; Tel: +44 (0)141 5484700

$\dagger$ Electronic supplementary information (ESI) available: Synthesis, characterisation, UV and membrane lysis experiments. See DOI: 10.1039/c5ob00465a
}

way of designing new photodynamic therapeutic agents for infectious diseases.

\section{Results and discussion}

Scheme 1 outlines the approach taken to merge the desired structural features of $\mathbf{1}$ and $\mathbf{2}$ and the subsequent synthesis of the target compound $(\mathbf{4}, 73 \%)$ using a TEMPO/CuBr-based oxidative coupling of $4-n$-octylaniline (3). ${ }^{10}$ With suitable quantities of the lipophilic azobenzene derivative $\mathbf{4}$ in hand, attempts to determine its spectroscopic and conformational properties were undertaken, prior to studying the interaction of any isolable conformers with phospholipid membranes.

To determine the response of 4 to UV light, a $0.125 \mathrm{mM}$ stock solution was prepared in a suitable solvent or water: solvent mix and spectra recorded every $10 \mathrm{~s}$ using $365 \mathrm{~nm}$ irradiation ( $\left.\mathrm{ESI}^{\dagger}\right)$. In the absence of UV irradiation, an intense $\pi-\pi^{*}$ transition band at around $330 \mathrm{~nm}$ and a weaker $\mathrm{n}-\pi^{*}$ transition band around $440 \mathrm{~nm}$ is observed for trans-4. ${ }^{3}$

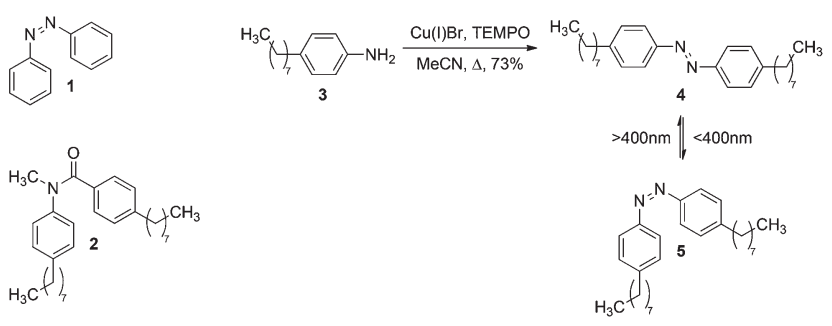

Scheme 1 Azobenzene (1), a lipid-interactive benzanilide (2) and the conformationally similar azobenzene derivatives studied here (4 and 5 ), and their synthesis. 
Following irradiation of 4 with $365 \mathrm{~nm}$ light for $180 \mathrm{~s}$ a spectrum indicative of $c i s-\mathbf{5}$ was recorded which possessed a $n-\pi^{*}$ transition with a higher intensity compared to the transisomer. ${ }^{3}$ Despite this difference, a photostationary equilibrium is set up meaning that a maximum of $\sim 85 \%$ of the cis-isomer forms upon irradiation at $330 \mathrm{~nm}$, an observation that was confirmed by ${ }^{1} \mathrm{H}$ NMR (see below).

To ensure that the observed conformational change was fully reversible, a relaxation experiment was carried out, as above, using a $532 \mathrm{~nm}$ laser as the light source and the now "excited" sample of 5 ( $0.125 \mathrm{mM}$ solution). In this case, after irradiation with the $532 \mathrm{~nm}$ laser using $30 \mathrm{~s}$ intervals for $10 \mathrm{~min}$, an identical time-course spectral series was recorded to that of the original trans-sample (4), indicating that the $n$-octyl-functionalised azobenzene (4) was able to switch conformations, as anticipated. ${ }^{11}$

Corroboration of the light-induced conformational switch was achieved when the experiment was repeated in $\mathrm{CDCl}_{3}$ and the irradiated sample analysed directly by NMR and compared to the non-irradiated sample. Similar changes in the UV spectra to those seen with the other solvents above were obtained in $\mathrm{CDCl}_{3}$. The changes in the ${ }^{1} \mathrm{H}$ NMR of 4 were compared prior to irradiation, after $21 \mathrm{~h}$ of $365 \mathrm{~nm}$ irradiation, and after exposure of the irradiated sample to darkness and heat or $532 \mathrm{~nm}$ light (to promote relaxation back to trans-4). The spectra (ESI $\dagger$ ) show an expected upfield shift occurs upon irradiation of trans-4, ${ }^{12}$ giving a spectrum which is consistent with the cis-conformation (5). Similarly, a switch back to the trans-conformer (4) occurs with $532 \mathrm{~nm}$ light or at $60{ }^{\circ} \mathrm{C}$ in the dark. In all spectra a small amount of the other conformer is visible, consistent with the UV experiments above. Combined, these results support that a conformational switch occurs between trans-4 and cis-5, whereby it switches from trans to cis at $365 \mathrm{~nm}$ and switches back again at $532 \mathrm{~nm}$ (or $60^{\circ} \mathrm{C}$ in the dark).

Having confirmed that the azobenzene derivative 4 cleanly undergoes the expected conformational switch, and that its relaxation back to the trans-conformer is sufficiently slow $(>21 \mathrm{~h})$ to enable assays to take place on the individual conformers, attention turned to establish what, if any, interactions the two conformers have with phospholipid membranes. Moreover, if the two conformers were able to interact with phospholipid membranes, it could be determined whether the results mirror those of the benzanilide conformers of 2 studied previously, ${ }^{6,8}$ whereby the cis-conformer was shown to be more membrane interactive than the trans-conformer, presumably a consequence of both lipid chains inserting into, and disrupting the membrane, rather than predominantly only one of the trans-compound. ${ }^{6,8}$ Furthermore, the light-switching advantage of the azobenzene series over the $N$-methylation state "pseudo-switch" of the amide series means that the compounds could be optimised for photodynamic therapy applications, especially for topical treatments.

A concentration-dependent calcein-release assay was used to determine the extent to which the two conformers are able to lyse liposomes loaded with the fluorescent dye. ${ }^{13}$ Four inde- pendent single-lipid liposomes were used which were composed of dimyristoyl phosphatidylethanolamine (DMPE), dimyristoyl phosphatidylglycerol (DMPG), dimyristoyl phosphatidylserine (DMPS) and dimyristoyl phosphatidylcholine (DMPC), phospholipids which are the main constituents in, and can therefore act as simple membrane mimics of, Escherichia coli (DMPE) and Staphylococcus aureus (DMPG) ${ }^{14}$ or cancer cells (DMPS and DMPC). ${ }^{15}$ The total lipid concentration used in the assay was $100 \mu \mathrm{M}$, with a $\mathrm{P} / \mathrm{L}$ ratio ranging from $\sim 1: 4$ $(24 \mu \mathrm{M})$ to $\sim 1: 33(3 \mu \mathrm{M})$.

The results (Fig. 1) demonstrate that the compounds do interact with phospholipid membranes, and that there is a significant difference between the two conformers, with cis-5 being more membrane-interactive than trans $\mathbf{4}$ across all lipids tested, results which mimic the trend of the benzanilide analogue too. ${ }^{6,8}$

The maximal lysis observed occurred with cis-5 and liposomes consisting of DMPS, where a plateau was reached at $12 \mu \mathrm{M}(\sim 64 \%$ lysis $)$ in $1 \mathrm{~h}$, a value that was not superseded even at double that concentration. These results compare well to those of cis- and trans-benzanilide $(2)^{6}$ against DMPE and DMPG liposomes where, even at the higher concentration of $400 \mu \mathrm{M}$, the maximum levels of lysis were $25.0 \pm 0.3 \%$ and $42.9 \pm 0.1 \%$ for the trans- and cis-conformers respectively against liposomes composed of DMPE lipid, and $21.1 \pm 0.6 \%$ and $41.9 \pm 0.2 \%$ for the trans- and cis-conformers respectively against liposomes composed of DMPG lipid. These can be compared to the data shown in Fig. 1 (at $12 \mu \mathrm{M}$ ) of $10.41 \pm$ $0.30 \%$ and $33.44 \pm 0.22 \%$ for trans -4 and cis-5 respectively against liposomes composed of DMPE lipid, and $28.58 \pm$
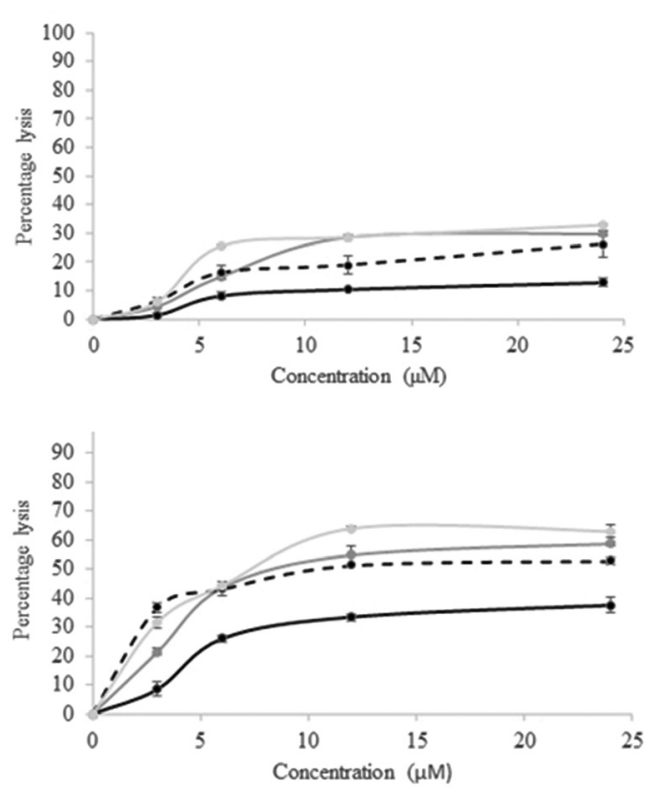

Fig. 1 The ability of trans-4 (top) and cis-5 (bottom) to induce membrane leakage using the lipids DMPE (solid black line), DMPG (solid dark grey line), DMPS (solid light grey line) and DMPC (dotted black line). The data shown are the average of three independent experiments. 
$0.21 \%$ and $54.72 \pm 0.31 \%$ for trans $-\mathbf{4}$ and cis-5 respectively against liposomes composed of DMPG lipid, suggesting that, in both cases, the cis-conformer is the most active form at lysing liposomes. Presumably such a trend occurs because in the cis-conformation the lipid chains are able to insert into the lipid bilayer and disrupt the membrane packing. Since conversion of the isomers at or near the membrane is the core concept here, the behaviour of the isomers in the presence of lipids was ascertained (ESI $\dagger$ ). A simple UV-Vis experiment was performed in which a spectral series of the azobenzene derivatives were followed over time in the presence of the liposomes made up from both DMPC and DMPE, and it was shown that identical series were obtained, in each case, to those of the series in the absence of the lipids, suggesting that the azobenzene derivatives are able to switch their conformations in the presence of lipids, as required. Since compounds 2, 4 and 5 contain the same lipid side-chains, the differences observed between the compounds and different liposomes studied is therefore seemingly dependent on the specific interactions between the phosphate headgroups and the motif linking the two adorned benzene rings. Although it is known that hydrophobic molecules can disrupt phospholipid membranes, there is a significant difference between trans-4 and cis-5 suggesting that shape plays an important role here too.

As can be seen in Fig. 2, the size of the two conformers varies such that the distance between the para-positions of the benzene rings (the location of the alkyl chains) is smallest in the cis-isomer $(\sim 0.5 \mathrm{~nm} c f$. $\sim 1.0 \mathrm{~nm})$. This geometry means that in the cis-conformation, the $n$-octyl chains are better positioned to interact with, and disrupt, the membrane packing (Fig. 2, left - shown for clarity with one molecule, although in reality clustering of the compounds in the membrane would most likely occur). Combined with the larger dipole moment of the cis-isomer enabling larger ion-dipole interactions to take place between 5 and the negatively charged membrane, makes the cis-isomer more membrane disruptive than the trans.

Whether studying the azobenzene derivative here, benzanilides ${ }^{6,8}$ ureas, $^{7}$ or other constructs, ${ }^{16,17}$ the subtle differences in the non-covalent interactions experienced between the particular molecular motif and the lipid headgroup should affect the stability of the compound in the membrane, and hence the level of lysis observed. ${ }^{18-24}$ For example, the membrane's charge varies depending the nature of the phosphate head-
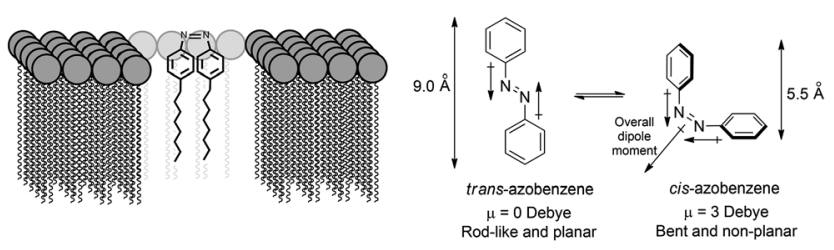

Fig. 2 (a) A schematic showing the proposed mechanism for membrane disruption of cis-5 (left, shown, for clarity with one molecule), and (b) the reversible isomerisation of azobenzene showing its geometry and dipole changes (right). group (i.e. ethanolamine, glycerol, serine and choline in DMPE, DMPG, DMPS and DMPC, respectively), as does the exact make-up of the charge, i.e. whether is it net neutral (zwitterionic) as seen in DMPE and DMPC, negative as seen in DMPG, or net negative (zwitterionic) as seen in DMPS, and this makes a difference in the levels to which the membrane is lysed. ${ }^{18,19}$

Such findings demonstrate that the properties of the lipid polar headgroup are important in such systems and the overall amount of lysis is governed by a fine balance of interaction contributions.

Having observed that the cis-conformer is more membraneinteractive than trans-4 over a $1 \mathrm{~h}$ time period, we were interested in understanding what would happen over an extended time period of $24 \mathrm{~h}$. This could be important since this is the sort of time-scale in which a cis $\rightarrow$ trans relaxation may begin to take place, and thus if it did whilst the compound was buried in the membrane, it would further increase the levels of membrane lysis observed, and its potential as a membranedisruptive compound.

Again, a concentration-dependent time course was studied against all four lipids (ESI $\dagger$ ), but representative data can be seen in Fig. 3 at $12 \mu \mathrm{M}$ for simplicity.

As seen in Fig. 3, against all lipids the cis-conformer (5) is more membrane lytic than trans-4, sometimes as much as twofold or more, and in most cases, maximal lysis occurs, and a plateau is reached, around the $3 \mathrm{~h}$ time-point. Overall maximal lysis occurs at $\sim 80 \%$ for cis-5 against DMPS, but interestingly,
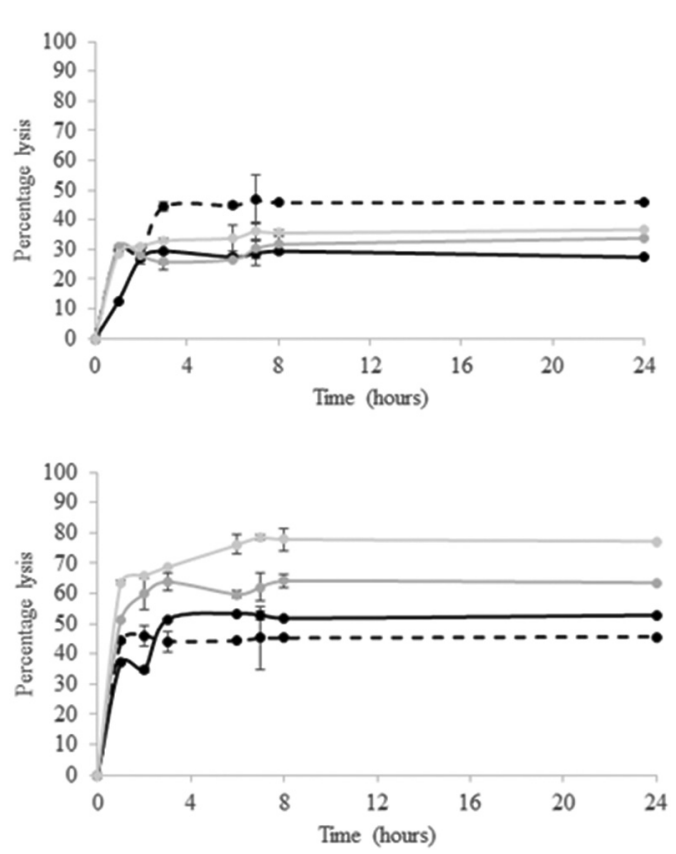

Fig. 3 The kinetics of calcein release over time after addition of $12 \mu \mathrm{M}$ $(\mathrm{P} / \mathrm{L}=\sim 1: 8)$ trans-4 (top) and cis-5 (bottom) to $100 \mu \mathrm{M}$ DMPE (solid black line), DMPG (dark grey line), DMPC (dotted black line) and DMPS (light grey line). After 7 hours the sample ceased calcein release and this did not change over time. 
there appears to be very little, if any, selectivity for liposomes composed of DMPC between the two conformers. If the difference between the levels of lysis caused by the cis and trans conformers for each time point are calculated, then it can be seen that, for all cases except the $1 \mathrm{~h}$ time-point, both the neutral (zwitterionic) lipids DMPE and DMPC have the smallest difference in lysis for the two conformers. This is in contrast to the two negatively charged lipids DMPG and DMPS, which have the greatest difference, and more specifically, the negative (zwitterionic) lipid DMPS is the most lysed liposome of all those studied. This difference provides a handle with which to potentially prepare compounds which can be photochemically switched to selectively interact with different phospholipid membranes, and thus be optimised to be selective chemotherapeutic agents.

Evidently, these subtle differences in phospholipid headgroups are able to impart small selectivities in the interaction of $\mathbf{4}$ and $\mathbf{5}$ with the different liposomes. However, the timepoint of the plateaus $(\sim 3 \mathrm{~h})$ are not long enough to induce a cis $\rightarrow$ trans transition under ambient conditions, and so we propose that the increased membrane lysis in the case of cis-5 is due to this conformer being able to better interact with the membrane than trans-4 (Fig. 1) and not due to isomerisation whilst embedded in the membrane, or hydrophobic effects alone.

\section{Conclusions}

In conclusion, we have shown that an azobenzene motif adorned with suitable $n$-octyl chains is able to disrupt a variety of independent calcein-loaded liposomes composed of four different phospholipids, and that the levels of lysis observed are dependent both on the lipid headgroup and the conformation of the molecules being studied. In all cases, it has been shown that the cis-conformer is more membrane-interactive than the corresponding trans-conformer, results which mimic those of other structurally-related conformational switches, ${ }^{6,8}$ suggesting that this class of molecule could be optimised for photo-dynamic therapy applications.

Future work will look to focus on studying mixed lipid systems and further adorning the benzene rings with watersolubilising groups, groups which could also shift the wavelength of light required to implement the conformational switch to the visible spectrum, ${ }^{1}$ as well as introducing charge to the molecules to increase their interaction with net negative membranes, as has seen success recently with pyridinium analogues. $^{9}$

\section{Acknowledgements}

The authors would like to thank the EPSRC UK National Mass Spectrometry Facility (NMSF), Swansea.

\section{Notes and references}

1 A. A. Beharry, O. Sadovski and G. A. Woolley, J. Am. Chem. Soc., 2011, 133, 19684-19687.

2 X. Wang, S. Werner, T. Weiss, K. Liefeith and C. Hoffmann, RSC Adv., 2012, 2, 156-160.

3 A. A. Beharry and G. A. Woolley, Chem. Soc. Rev., 2011, 40, 4422-4437.

4 M. Banghart, K. Borges, E. Isacoff, D. Trauner and R. H. Kramer, Nat. Neurosci., 2004, 7, 1381-1386.

5 M. J. Hansen, W. A. Velema, G. de Bruin, H. S. Overkleeft, W. Szymanski and B. L. Feringa, ChemBioChem, 2014, 15, 2053-2057.

6 S. R. Dennison, Z. Akbar, D. A. Phoenix and T. J. Snape, Soft Matter, 2012, 8, 3258-3264.

7 S. R. Dennison, D. A. Phoenix and T. J. Snape, Bioorg. Med. Chem. Lett., 2013, 23, 2518-2521.

8 S. R. Dennison, T. J. Snape and D. A. Phoenix, Eur. Biophys. J., 2012, 41, 687-693.

9 S. Fahs, F. B. Rowther, S. R. Dennison, Y. Patil-Sen, T. Warr and T. J. Snape, Bioorg. Med. Chem. Lett., 2014, 24, 34303433.

$10 \mathrm{Z} . \mathrm{Hu}$ and F. M. Kerton, Org. Biomol. Chem., 2012, 10, 1618-1624.

11 S. Moeller, U. Pliquett and C. Hoffmann, RSC Adv., 2012, 2, 4792-4801.

12 K. M. Tait, J. A. Parkinson, A. C. Jones, W. J. Ebenezer and S. P. Bates, Chem. Phys. Lett., 2003, 374, 372-380.

13 T. M. Allen and L. G. Cleland, Biochim. Biophys. Acta, 1980, 597, 418-426.

14 K. Lohner and E. J. Prenner, Biochim. Biophys. Acta, 1999, 1462, 141-156.

15 T. Utsugi, A. J. Schroit, J. Connor, C. D. Bucana and I. J. Fidler, Cancer Res., 1991, 51, 3062-3066.

16 A. D. Bautista, C. J. Craig, E. A. Harker and A. Schepartz, Curr. Opin. Chem. Biol., 2007, 11, 685-692.

17 A. Som, S. Vemparala, I. Ivanov and G. N. Tew, Biopolymers, 2008, 90, 83-93.

18 A. A. Stromstedt, P. Wessman, L. Ringstad, K. Edwards and M. Malmsten, J. Colloid Interface Sci., 2007, 311, 59-69.

19 T. Le Bihan, D. Pelletier, P. Tancrede, B. Heppell, J. P. Chauvet and C. R. Gicquaud, J. Colloid Interface Sci., 2005, 288, 88-96.

20 R. M. Epand, Biochim. Biophys. Acta, 1998, 1376, 353-368.

21 R. M. Epand and R. F. Epand, Biochim. Biophys. Acta, 2009, 1788, 289-294.

22 R. M. Epand and R. F. Epand, J. Pept. Sci., 2011, 17, 298305.

23 B. P. Mowery, S. E. Lee, D. A. Kissounko, R. F. Epand, R. M. Epand, B. Weisblum, S. S. Stahl and S. H. Gellman, J. Am. Chem. Soc., 2007, 129, 15474-15476.

24 D. Koller and K. Lohner, Biochim. Biophys. Acta, 2014, 1838, 2250-2259. 